\title{
Psychological approaches to somatisation in developing countries
}

\author{
Vikram Patel \& Athula Sumathipala
}

\begin{abstract}
Medically unexplained somatic complaints are among the most common clinical presentations in primary care in developing countries and they are a considerable burden for patients and the healthcare system. They are assumed to be the result of psychosocial factors, and the process by which symptoms are experienced is termed somatisation. Common mental disorders, somatoform disorders and socioeconomic adversities are the major risk factors for these complaints. There is evidence suggesting that cognitive-behavioural therapy, which has proven efficacy for somatoform disorders in the developed world, can be used in developing countries with some adaptations (e.g. by simplifying the content so that it can be applied in primary care by non-specialist health practitioners; using culturally appropriate analogies; and delivering the intervention over fewer and shorter sessions). The main components of such an intervention are presented in this article.
\end{abstract}

Somatic complaints that cannot be attributed to a physical illness are described as 'medically unexplained' or 'functional', and it is assumed that psychological factors are their primary cause. Medically unexplained somatic symptoms have been classified in many different ways, for example according to the duration of symptoms (acute or chronic) or their location in the body (for example, irritable bowel syndrome, atypical chest pain). The precise psychological basis for medically unexplained symptoms remains debatable. About half of patients with medically unexplained symptoms meet criteria for mood and anxiety disorders (Kroenke et al, 1994; Simon et al, 1996). The rest fall into the ICD-10 (World Health Organization, 1992) and DSM-IV (American Psychiatric Association, 1994) diagnostic category of somatoform disorders, which are characterised by

'the presence of physical symptoms that suggest a general medical condition ... a and are not fully explained by a general medical condition, by the direct effects of a substance, or by another mental disorder' (American Psychiatric Association, 1994: p. 445).

However, the clinical usefulness of these diagnostic categories for understanding medically unexplained symptoms has been questioned. Bass \& Benjamin (1993) conceptualise somatisation as a process, rather than a diagnosis, in which the individual has an inappropriate focus on physical symptoms and psychosocial problems are denied. According to Kleinman \& Kleinman (1985)

'somatisation is the expression of personal and social distress in an idiom of bodily complaints with medical help seeking' (p. 430).

Somatisation, in this context, is not a disease entity but a process whose result is the illness experience of medically unexplained symptoms.

It was often assumed that somatisation was a characteristic of mental disorder in non-Western societies and that this was because non-Western cultures were less accepting of psychological symptoms and mental illness. However, it is now acknowledged that somatic presentations are characteristic of all cultures and, furthermore, that the psychological symptoms of common mental disorders can often be elicited on enquiry (Patel et al, 1998; Araya et al, 2001). Thus, somatisation is a universal phenomenon; indeed, some authors have argued that it is 'psychologisation', the presentation of common mental disorders as psychological symptoms, that is the more unusual presentation (Goldberg \& Bridges, 1988). The most likely explanation for somatisation is that somatic complaints are a direct consequence of common mental disorders; for example, autonomic symptoms may be the result of hyperventilation as a consequence

Vikram Patel is a reader in international mental health at the London School of Hygiene and Tropical Medicine (NPHIRU, London School of Hygiene and Tropical Medicine, Keppel Street, London WC1E 7HT, UK. e-mail: Vikram.patel@lshtm.ac.uk). Athula Sumathipala is an honorary lecturer at the Institute of Psychiatry, London. Both authors are psychiatrists who have been working in South Asia, conducting research into public health aspects of mental disorders, particularly the efficacy and cost-effectiveness of locally available and feasible treatments in primary care, and building research capacity in the region. Both wish to acknowledge the Wellcome Trust for supporting their research on mental disorders in South Asia. 
of anxiety. In addition, somatic complaints may be idioms for help-seeking for severe social adversities such as domestic violence. Other reasons for somatisation include the stigma associated with mental illness and the perception (often based on reality) that doctors are more concerned with, and likely to respond to, somatic symptoms.

In this article we use the term 'somatic complaints' to denote physical complaints that are clearly not attributable to a physical disease, and focus our discussion of management on symptoms associated with somatoform disorders.

\section{Somatic complaints in developing countries}

\section{Clinical presentation}

There has been limited research on the clinical presentation of somatic symptoms (Chaturvedi et al, 1988; Chaturvedi et al, 1993; Isaac et al, 1995; Janca et al, 1995; Sumathipala et al, 2004) and the prevalence and aetiology of somatic complaints in primary care in developing countries (Gureje et al, 1997). The key finding of these studies is that, despite the enormous variation in the prevalence of various somatic complaints, the overall burden of such complaints is high. Some complaints are universal, being described in developed countries as well. Prominent among these are: fatigue and tiredness; aches and pains, notably headaches and generalised body pains; and abdominal discomfort. Some somatic syndromes, such as the loss of semen syndrome, were once widely recognised in developed societies, but are now largely found only in non-Western cultures (Sumathipala et al, 2004). These historical trends are perhaps best understood in the context of Cartesian dualism, which became a strong influence on Western biomedical concepts in the early 20 th century.

\section{Cultural context}

Some complaints appear to occur within specific cultural contexts. Cultural factors may influence the way a somatic complaint is described. For example, what might be described as tingling-numbness in one culture may be described as insects crawling on the skin in another. A number of somatic symptoms, especially those related to the heart and to the head, prove to be rich cultural metaphors for fear or grief (Patel, 1995). Cultural factors may also influence the way somatic symptoms are categorised. Perhaps the best known example is neurasthenia, a syndrome characterised by fatigue and other somatic symptoms, which is now diagnosed almost exclusively in East Asia. It seems that fatigue, the defining symptom of neurasthenia, is a culturally sanctioned expression of distress, not only of psychological distress such as depression, but also of social unease (Kleinman \& Kleinman, 1985). Finally, cultural factors may influence the 'choice' of somatic complaint, for example the complaint of vaginal discharge in women in South Asia (Patel et al, 2005b).

\section{Aetiology}

The importance of the aetiology of somatic complaints in developing countries is underscored by the fact that the epidemiological pattern of the burden of disease is very different. For example, the complaint of fatigue is typically attributed to nutritional deficiencies and, in particular, anaemia, which is common in women on account of menstrual blood loss and poorer nutrition, child-bearing and multiple pregnancies (Lennartsson et al, 1979). As a consequence, physicians are likely to prescribe iron, vitamins, tonics and other nutritional supplements to treat the symptom presumptively. However, this response has been challenged by a recent community study in India that sheds light on the prevalence and risk factors for the complaint of chronic fatigue in women (Patel et al, 2005a). More than 1 in 10 women reported fatigue of at least 6 months' duration. The strongest risk factors were socio-economic deprivation, gender disadvantage and poor mental health (Box 1). There was no association between haemoglobin levels or frank anaemia and the complaint of chronic fatigue.

\section{Epidemiological conclusions}

Thus, despite widely varying cultural beliefs and epidemiological profiles of disease burden in developing countries, the epidemiology of somatic complaints in these countries is very similar to that in developed countries; the key difference is the

Box 1 Strongest risk factors for the complaint of chronic fatigue in women in India

- Socio-economic deprivation - poor education, being in debt and having experienced hunger in the recent past

- Gender disadvantage - widowhood, oppressive restrictions on women's lives, lack of a trusting relationship with a spouse, and marital violence

- Poor mental health-comorbidity with other physical complaints, and symptoms of depression and anxiety 
nature of the social adversities and the types of illness model that might predispose individuals to or precipitate these complaints. The management of somatic symptoms should address their psychosocial risk factors.

\section{The psychological management of somatic symptoms}

A comprehensive literature search using Medline and PsycInfo identified a number of trials of psychological treatments carried out in primary, secondary and tertiary care. However, only one trial, by Sumathipala et al (2000) in Sri Lanka, was identified from a developing country. Consequently, we present the findings of our review in two parts. First, we summarise the evidence base for the effectiveness of psychological interventions for somatisation in developed countries. Second, we consider how this evidence base has been applied in Sri Lanka, focusing on the practical clinical steps suitable for the sociocultural characteristics of the setting.

\section{The evidence base from developed countries}

Our search identified trials using problem-solving therapy, cognitive therapy, cognitive-behavioural therapy, reattribution training and brief dynamic psychotherapy for the management of functional somatic symptoms; the most frequently used for this purpose is cognitive-behavioural therapy.

\section{Cognitive-behavioural therapy}

Most of the trials we assessed give short-term outcomes only. Although medically unexplained symptoms are mainly seen in primary care settings, most of the trials were carried out in secondary or specialist care. A number of systematic reviews evaluated the use of cognitive-behavioural therapy in the treatment of people with somatic symptoms (e.g. Kroenke \& Swindle, 2000; Looper \& Kirmayer, 2002). Most of the trials targeted specific syndromes such as irritable bowel or chronic fatigue syndromes. Thus, only a minority of trials specifically focused on general somatisation, which includes people with medically unexplained symptoms and those with hypochondriasis.

The reviews conclude that cognitive-behavioural therapy, delivered in individual or group format, may be efficacious for somatic symptoms whether defined as symptom syndromes or grouped under the broader headings of somatoform disorders. Trials variously found that cognitive-behavioural therapy reduced physical symptoms, psychological distress and disability. Cognitive-behavioural interventions produced effects of moderate to large magnitude and cognitive-behavioural therapy is recommended as the first line of treatment. However, the optimum and minimum duration of treatment and the value of maintenance therapy remain to be established.

\section{Psychodynamic psychotherapy}

A systematic review of psychodynamic psychotherapy (Guthrie, 1996) identified three trials, from which it concluded that psychodynamic psychotherapy was effective for the management of chronic pain and irritable bowel syndrome. However, the small number of empirical studies made it difficult to generalise the results to other somatic conditions.

\section{Reattribution training}

A trial of reattribution training reported that it was associated with more frequent endorsement by patients that they had received the help they wanted and fewer beliefs that their symptoms had only a physical cause. However, the training did not change the incidence of investigations initiated by the family doctor, prescriptions for psychotropic or non-psychotropic drugs or referrals (Morriss \& Gask, 2002).

\section{Patient-practitioner interaction}

Apart from specific psychological treatments, there is a growing body of evidence that patient-health practitioner interaction may have benefits for patients with somatoform disorders. For example, Price (2000) noted that an assessment for psychotherapy might itself have therapeutic effects. Changing patients' beliefs about their symptoms may improve a broad range of outcomes, including the symptoms themselves, disability, distress and use of healthcare services. Petrie et al (1995) reported that a rounded clinical assessment might modify cognitive factors such as symptom attribution and improve outcome. Price (2000) concluded that simple cognitive approaches might be able to change cognitions and make meaningful improvements in outcomes. This approach acknowledges that symptoms might have a pathophysiological and a cognitive basis. There is evidence from randomised trials to support the therapeutic effect of explanation, supported only by history-taking and consultation. For example, a consultation in which a patient is given a definite diagnosis and reassurance that they will be better soon is better than one in which they are told that their diagnosis 
and outcome are uncertain (Thomas, 1987). There is also evidence that the treatment of patients presenting with somatic complaints by general physicians rather than psychiatrists leads not only to improved clinical outcomes, but is also associated with improved physical functioning and reduced healthcare costs (Smith et al, 1995).

\section{The evidence base in developing countries}

The strongest evidence base from developed countries is for cognitive-behavioural therapy. A key problem in translating this evidence crossculturally is the assumption that the underlying cognitions are essentially similar in Western and non-Western cultures and that the concepts underpinning cognitive-behavioural therapy will be valid in different cultural settings. As mentioned above, we are aware of only one published treatment trial for medically unexplained symptoms from developing countries (Sumathipala et al, 2000) and this trial used a cognitive-behavioural approach.

\section{Cognitive-behavioural therapy in Sri Lanka}

Primary care attenders at a general out-patient clinic in Sri Lanka who had five or more medically unexplained complaints were randomised to cognitive-behavioural therapy with a psychiatrist or to treatment as usual. Cognitive-behavioural therapy reduced patients' distress and symptoms, decreased the number of patient-initiated visits to a doctor and increased patient satisfaction. Three 30-min sessions were found to be the minimum adequate course of treatment. A reduction in symptoms was also found in the control group (routine primary care). The researchers concluded that the opportunity to participate in a detailed assessment and to express their explanatory model of illness ${ }^{1}$ had a non-specific therapeutic effect.

\section{Cognitive representation of illness}

The 'cognitive representation of illness' model (Horne, 1997) describes how an individual constructs an internal representation of what is happening to them when they experience physical or psychological symptoms. It suggests that, no matter what the nature of the symptoms, most people organise their thinking around the five key themes of identity, cause, timeline, consequences

1. Explanatory models have been discussed in an earlier issue of $A P T$ : see Bhui \& Bhugra (2004) Communication with patients from other cultures: the place of explanatory models. Advances in Psychiatric Treatment, 10, 474-478. Ed.
Box 2 The five themes of the cognitive representation of illness model

- What is it? (identity)

- Why has it happened? (cause)

- How long will it last and will it recur? (timeline)

- What effects will it have? (consequences)

- What can I do to make it go away? (cure/ control)

and cure/control. These themes are clarified in Box 2.

In terms of this model, the majority of patients in the Sri Lankan trial were not concerned about the identity of their symptoms or their cause; they were mainly concerned only about the timeline, consequences and cure/control. The experiences from this trial suggest that the theoretical model underpinning cognitive-behavioural therapy does have cross-cultural applicability, although (as described below) the specific details of the intervention required modification to suit the local context.

\section{Intervention in primary care}

Given the great shortage of mental health professionals in developing countries, trials of psychological treatments for use by non-mental health specialists are of special interest. One such is an evaluation of the effect of a teaching package of reattribution therapy for general practice trainees in the UK and in Tanzania (Kaaya et al, 1992). Although this study showed that skills could be transferred to general practitioners, there was weak evidence that the intervention led to significant improvement in clinical outcomes.

\section{Psychological management in developing countries}

People presenting with multiple somatic symptoms are a heterogeneous group, some with an underlying common mental disorder, others with an unrelated medical illness and some with neither (Bass \& Benjamin, 1993; Wessely, 1996). We will not consider here the specific management of common mental disorders.

Clinical management of somatic complaints should aim not only to improve the clinical outcome for the patient and family, but also to minimise the direct and indirect disproportionate healthcare costs incurred by patients with somatoform disorders. In both developed and developing 
countries individuals with somatic complaints tend to consult specialist and alternative care providers (Sumathipala, 1990; Sharpe \& Carson, 2001). However, they seem to dislike psychiatric referral, and it rarely results in effective treatment (Escobar, 1996; Mayou \& Sharpe, 1997). Furthermore, the great scarcity of mental health services in developing countries and the considerable stigma associated with the seeking of mental healthcare emphasise that intervention must be delivered in primary care by a community or primary health practitioner. The aim of the chosen intervention should be to 'contain' the patient at the primary care level, by offering regular structured visits to one professional carer, thereby coordinating the care and, hopefully, reducing patient-initiated 'unstructured' visits to different practitioners. Through structured sessions, individuals can be made aware of the psychological component of their condition and helped to reduce unnecessary medical consultations and investigations. The ultimate goal of the treatment should be to reduce the patient's distress, symptoms and disability and to reduce or limit inappropriate use of medical services.

\section{A psychological treatment: key elements}

In this section we summarise some of the key elements of a psychological treatment approach. The specific approach we describe is adapted from the manual used by one of us (A.S.) with colleagues in a recent trial in Sri Lanka of an intervention delivered by primary care physicians to patients with somatic complaints. The treatment was based on a model of cognitive-behavioural therapy described in an earlier trial (Sumathipala et al, 2000), which adapted methods developed by Salkovskis (1989), Sharpe et al (1992) and Goldberg et al (1989). The adaptations included innovative use of culturally relevant and appropriate psychotherapeutic language and strategies simple enough for use in primary care, but conforming to cognitivebehavioural principles.

The trial involved individuals whose symptoms had been present for more than 6 months (to exclude those with acute somatic complaints who were likely to recover spontaneously). The cognitive-behavioural intervention was administered by primary care physicians. Patients were assessed by a consultant physician at baseline and at 3, 6, 9 and 12 months to identify the number of complaints and visits (after the initial treatment sessions all visits were patient-initiated) at each assessment. Participants in the control groups were also cared for by designated primary care physicians, who offered the same number of
Box 3 The key elements of a psychological approach

- Recapitulation of the problem

- Acknowledging that the symptoms, distress and disability are genuine

- Explaining strategy of treatment

- Limiting help-seeking

- Explaining the nature of somatic complaints

- Concentrating on patient's explanatory models

- Avoiding unnecessary treatment and investigations

- Encouraging return to normal activities

- Diary-keeping and monitoring of progress

contacts received by the intervention groups, thus controlling for any non-specific therapeutic effect of the intervention. The first three sessions of the intervention were mandatory and weekly. The remaining three were optional and fortnightly. Each session lasted for $30 \mathrm{~min}$ and the treatment was spread over 9 weeks. The intervention was based on the following key elements (summarised in Box 3).

\section{Recapitulation of the problem}

The first step is recapitulation of the problem using the information obtained during exploration of the patients' explanatory model of their illness. This is an important element because it tells the patient that they have been understood, that their distress has been appreciated as genuine and not imagined and that the practitioner can empathise with it. This helps establish rapport, which will eventually lead to a strategic alliance and a paradigm shift from what they might have experienced so far in a protracted journey for relief. At this stage of the treatment it is also important to avoid any suggestion that psychological factors or psychiatric illness play a role. The practitioner should recapitulate the problem and present a summary such as:

\footnotetext{
'You've come to me today as you've had abdominal pain, headache, chest pain, backache, pain in your right arm and numbness in your fingers for the past 5 years. You can't give an exact name for your illness but believe that working too much caused it. You also feel that your husband is responsible for it, as he never helped you with the housework. You believe that your illness is very serious and suspect it might be cancer. You've been to eight different doctors and had an ECG, chest and spine X-rays, blood tests, urine tests and many other tests you can't describe. All of those were normal. Most of the doctors told
} 
you that there was nothing wrong. However, you are far from happy as the symptoms persist, and you strongly feel that you should have a full-body CT scan. As a result of the symptoms, you're unable to do any housework and have had to quit your permanent job as a cashier.'

Acknowledging that the symptoms, distress and disability are genuine

The practitioner should state clearly that they believe the patient's complaints and concerns to be genuine and not a sign of malingering. They should then explain that any symptom, irrespective of its cause, can make people worry. This could be phrased as follows:

'It's wrong to say that there is nothing wrong. There is something wrong. We will help you find out what it is and guide you to do what is best. We will also try to persuade you to avoid doing harmful things. More importantly, I must tell you that your symptoms do not mean that you are going to die or be permanently disabled. I also assure you that you are not 'mad', the symptoms are not 'all in your mind' and I know that you're not telling lies.'

\section{Explaining the treatment strategy}

Next, the practitioner outlines the planned treatment and explains its objectives (Box 4). The patient is made aware of their own role in making the treatment successful, i.e. the importance of their responsibility for taking control of their condition. They are requested to enter into a verbal contract on the treatment:

'It is very important for you to actively take part in the treatment process. I can help you do it but I cannot do it for you. Obviously you can't do it all by yourself and that's why you're here today. I will help you get over the difficult situation you are now in. Your treatment is, in fact, a partnership in which I can teach you what to do, how to do it and what things you should not do. This will enable you to deal with your symptoms yourself instead of depending too much on medical help, which hasn't done a great job for you so far.

Let me explain a bit more about the nature of the treatment we would offer you. You'll already have noticed that we spend more time with you here than the doctors you've seen so far, and we also talk more. So, for now, let's call it 'talking treatment'. This treatment is commonly used in other parts of the world. It enables the doctor and the patient to talk not only about the patient's symptoms but also about the distress that these cause. It also helps both of us to understand why all these things are happening. So we can exchange and share our views and plan and work together to get over these difficulties. Over the next six sessions you'll learn the dos and don'ts.'

\section{Box 4 The ultimate goal of treatment}

- To reduce the patient's distress, symptoms and disability

- To reduce or limit inappropriate use of medical services and medication

The practitioner should also give the patient a handout written in simple language indicating the contents of the first sessions. This should include the treatment goals listed in Box 4 and what the patient is expected to do and not do during the treatment session.

\section{Limiting help-seeking}

The practitioner should reassure the patient that if visits are required to any other specialist, the practitioner will arrange them in a coordinated fashion. In addition to getting the patient to agree to work with one professional carer, it is useful to engage only one non-professional carer (for example, the spouse or a family member). The aim is to train this person to be a 'co-therapist', who can reinforce over the long term the treatment offered in the brief therapeutic sessions. The cotherapist's main strategy is to try to discourage the patient from discussing symptoms and worries with different people at different times, as conflicting explanations and advice may add to the confusion. The patient is instructed not to discuss symptoms with anyone except their 'co-therapist', who is learning how to help them.

\section{Explaining the nature of somatic complaints}

The practitioner should explain in simple language the basis of perception of symptoms, saying for example:

'Various perceptions or feelings in our bodies are common and, regardless of whether we do something about them or ignore them, in most instances they wane in time. If they persist, we may get more alarmed and often, even without being aware of it, we may start to guess the significance of these symptoms. This can lead to fear, distress, concern or worry, an experience that is often shaped by our own previous episodes of illness or those of our relatives. The more concerned we become, the more vigilant and hence the more we notice the symptoms that worried us in the first place and also things we hadn't noticed before.

If we listen hard we pick up faint noises. If we look carefully, we see things we wouldn't see otherwise. Similarly, if we're preoccupied with our own body we'll notice various sensations. For example, you breathe all the time but usually you're not aware of it. 
But if you close your eyes and concentrate on your breathing you'll feel it. So the more preoccupied you are with symptoms the more you'll notice.'

\section{Concentrating on the patient's explanatory model}

The explanatory model offers an alternative approach for understanding the continued distress of individuals with medically unexplained symptoms. The practitioner can construct the patient's explanatory model for their illness by using the information that the patient gives during the consultation (Box 5). This model will be helpful in the development of an appropriate intervention. The practitioner should discuss the patient's fears with them and provide appropriate reassurance. Any reasonable fears that have not been addressed should be dealt with accordingly. It is important to explain how other people's opinions and behaviours can adversely affect distress.

Avoiding unnecessary treatment and investigations

It is important to explain why no further symptomatic drug treatments will be encouraged, and why no more blood tests or laboratory tests are needed if the symptoms have already been investigated. The practitioner should also explain the reinforcing effect of unneeded investigations that have negative results. This should be discussed in relation to the lack of relevance of the investigation to the symptoms. If an irrelevant investigation has been carried out and the result is negative, then the doctor may say that there is nothing wrong. However, the patient knows that their symptoms are genuine and may therefore suspect that they have a serious illness which has not been detected by the doctor. This may lead to greater anxiety and to consultations with more doctors. Thus, paradoxically, negative test results can make patients more worried.

Box 5 Constructing the patient's explanatory model

Key clues revealing the patient's model:

- their assumptions

- beliefs

- thoughts about their illness and its causes

- associated fears

- the impact of their symptoms, especially reduction in usual functions and increase in help-seeking and utilisation of healthcare

- their expectations of treatment and recovery
The 'why is an elephant called an elephant?' analogy is useful in explaining why more investigations that are likely to lead to more negative findings are not needed. The practitioner draws a sketch of an elephant and asks the patient what it is. Assuming that the patient replies that it is an elephant, the practitioner asks why they say it is an elephant. The patient will then give reasons - it has a trunk, tusks and so on. The practitioner then replies:

'So, it is an elephant based on specific features of an elephant. It was not by excluding other animals that you came to the conclusion that it is an elephant. The elephant is an elephant not because it is not a cat, a cow, a rat ... or any other animal.'

\section{Encouraging a return to normal activities}

The disproportionate distress and disability experienced by patients with somatic complaints may result in the overinvolvement of the family. The patient may be relieved of their usual day-today responsibilities because of their perceived disability. However, this reinforces rather than resolves their beliefs and dysfunctional behaviours. Sometimes patients avoid an activity because they are afraid of provoking symptoms. Sometimes avoidance may be directly attributed to a symptom such as 'lack of energy'. Such behaviour may lead the individual to take time off work, to abandon domestic work or even withdraw from social encounters. Again, the resultant disability will reinforce the fear and concern of potential serious illness.

\section{Diary-keeping and monitoring progress}

Diary-keeping is a means of expressing distress and of identifying dysfunctional cognitions. A diary can also used as a basis for monitoring symptoms. From the first session the practitioner should encourage the patient to make regular notes of symptoms, associated thoughts (the cognitions) and related behaviour. They should be given a diary for this purpose. In subsequent sessions, the practitioner should go through the diary entries with the patient and use them to reinforce the teaching of previous sessions.

\section{Conclusions}

Medically unexplained somatic complaints are among the most common clinical presentations in primary care in developing countries; they cause considerable distress and disability in patients and are a burden to the healthcare system. In the 
developed world individuals with such complaints can be effectively treated using evidence-based principles for the psychological management of common mental disorders (Patel et al, 2004). Very limited experience reveals that psychological management is feasible and possibly effective in the developing world as well. Specifically, the cognitive-behavioural model has been shown to be adaptable and applicable in Sri Lanka.

Complex psychological principles can be translated into simple components that both patients and non-specialist 'therapists' can understand. The culturally appropriate analogies used in the therapeutic package described here retained the fundamentals of cognitive-behavioural theory. This cross-cultural comparability of the treatment model is important, as it strengthens its validity. Fewer and shorter sessions (compared with standard cognitive-behavioural therapy) delivered by a nonspecialist health practitioner achieved significant clinical improvement. This finding has implications not only in the developing world, where mental health services are scarce, but also in the developed world, where psychological therapy is expensive.

Education of healthcare professionals of all specialties and grades about the burden of medically unexplained symptoms is an important priority. A realistic aim in the management of this common clinical problem is to identify it early and to treat it at whatever level of care the patient presents. To achieve the target of identifying and treating these patients at primary care level, it is crucial to train primary care practitioners in the relevant skills.

\section{Declaration of interest}

None.

\section{References}

American Psychiatric Association (1994) Diagnostic and Statistical Manual of Mental Disorders (4th edn) (DSM-IV). Washington, DC: APA.

Araya, R., Rojas, G., Fritsch, R., et al (2001) Common mental disorders in Santiago, Chile. Prevalence and sociodemographic correlates. British Journal of Psychiatry, 178, 228233.

Bass, C. \& Benjamin, S. (1993) The management of chronic somatisation. British Journal of Psychiatry, 162, 472-480.

Chaturvedi, S., Upadhyaya, M. P. \& Rao, S. (1988) Somatic symptoms in a community clinic. Indian Journal of Psychiatry, 30, 369-374.

Chaturvedi, S., Chandra, P., Isaac, M. K., et al (1993) Somatization misattributed to non-pathological vaginal discharge. Journal of Psychosomatic Research, 17, 575-579.

Escobar, J. I. (1996) Overview of somatization: diagnosis, epidemiology, and management. Psychopharmacological Bulletin, 32, 589-596.

Goldberg, D. \& Bridges, K. (1988) Somatic presentations of psychiatric illness in primary care settings. Journal of Psychosomatic Research, 32, 137-144.
Goldberg, D. P., Gask, L. \& O'Dowd, T. (1989) The treatment of somatisation - Teaching techniques of reattribution. Journal of Psychosomatic Research, 33, 689-695.

Gureje, O., Simon, G. E., Ustun, T. B., et al (1997) Somatization in cross-cultural perspective: a World Health Organization study in primary care. American Journal of Psychiatry, 154, 989-995.

Guthrie, E. (1996) Psychotherapy for somatisation disorders. Current Opinion in Psychiatry, 9, 182-187.

Horne, R. (1997) Representations of medication and treatment: advances in theory and measurement. In Perceptions of Health and Illness (eds K. Petrie \& J. Weinman), pp. 142-154. London: Harwood Academic.

Isaac, M. K., Janca, A., Burke, K. C., et al (1995) Medically unexplained somatic symptoms in different cultures. A preliminary report from phase I of the World Health Organization International Study of Somatoform Disorders. Psychotherapy and Psychosomatics, 64, 88-93.

Janca, A., Isaac, M. K., Bennett, L. A., et al (1995) Somatoform disorders in different cultures - a mail questionnaire survey. Social Psychiatry and Psychiatric Epidemiology, 30, 44-48.

Kaaya, S., Goldberg, D. \& Gask, L. (1992) Management of somatic presentations of psychiatric illness in general medical settings: evaluation of a new training course for general practitioners. Medical Education, 26, 138-144.

Kleinman, A. \& Kleinman, J. (1985) Somatization: the interconnections in Chinese society among culture, depressive experiences, and the meanings of pain. In Culture and Depression (eds A. Kleinman \& B. Good), pp. 429-490. Berkeley, CA: University of California Press.

Kroenke, K. \& Swindle, R. (2000) Cognitive-behavioral therapy for somatization and symptom syndromes: a critical review of controlled clinical trials. Psychotherapy and Psychosomatics, 69, 205-215.

Kroenke, K., Spitzer, R. I., William, J. B., et al (1994) Physical symptoms in primary care. Predictors of psychiatric disorders and functional impairment. Archives of Family Medicine, 3, 744-749.

Lennartsson, J., Bengtsson, C., Hallberg, L., et al (1979) Characteristics of anaemic women. The population study of women in Goteborg 1968-1969. Scandinavian Journal of Haematology, 22, 17-24.

Looper, K. J. \& Kirmayer, L. J. (2002) Behavioral medicine approaches to somatoform disorders. Journal of Consulting and Clinical Psychology, 70, 810-827.

Mayou, R. \& Sharpe, M. (1997) Treating medically unexplained physical symptoms. Effective interventions are available. $B M J, 315,561-562$.

Morriss, R. K. \& Gask, L. (2002) Treatment of patients with somatized mental disorder: effects of reattribution training on outcomes under the direct control of the family doctor. Psychosomatics, 43, 394-399.

Patel, V. (1995) Explanatory models of mental illness in subSaharan Africa. Social Science and Medicine, 40, 1291-1298.

Patel, V., Pereira, J. \& Mann, A. (1998) Somatic and psychological models of common mental disorders in India. Psychological Medicine, 28, 135-143.

Patel, V., Araya, R. \& Bolton, P. (2004) Treating depression in developing countries. Tropical Medicine and International Health, 9, 539-541.

Patel, V., Kirkwood, B. R., Weiss, H., et al (2005a) Chronic fatigue in developing countries: population based survey of women in India. BMJ, 330, 1190-1193.

Patel, V., Pednekar, S., Weiss, H., et al (2005b) Why do women complain of vaginal discharge? A population survey of infectious and pyschosocial risk factors in a South Asian community. International Journal of Epidemiology, 34, 853-862.

Petrie, K., Moss-Morris, R. \& Weinman, J. (1995) The impact of catastrophic beliefs on functioning in chronic fatigue syndrome. Journal of Psychosomatic Research, 39, 31-37.

Price, J. (2000) Review: antidepressants are effective for clinical unexplained symptoms and syndromes. Evidence Based Mental Health, 3, 84.

Sharpe, M. \& Carson, A. (2001) Unexplained somatic symptoms, functional syndromes and somatisation. Do we need a paradigm shift? Annals of Internal Medicine, 134, 926-930. 
Sharpe, M., Peveler, R. \& Mayou, R. (1992) Invited review: The psychological treatment of patients with functional somatic symptoms. A practical guide. Journal of Psychosomatic Research, 36, 515-529.

Salkovskis, P. M. (1989) Somatic problems. In Cognitive Behaviour Therapy for Psychiatric Problems: A Practical Guide (eds K. Hawton, P. M. Salkovskis, J. Kirk, et al). Oxford: Oxford University Press.

Simon, G., Gater, R., Kisely, S., et al (1996) Somatic symptoms of distress. An international primary care study. Psychosomatic Medicine, 58, 481-488.

Smith, G. R., Rost, K. \& Kashner, M. (1995) A trial of the effect of a standardised psychiatric consultation on health outcome and costs in somatising patients. Archives of General Psychiatry, 52, 238-243.

Sumathipala, A. (1990) Psychiatric Disturbance in Patients with Multiple Complaints and/or Repeated Consultations. MD thesis. Colombo: Postgraduate Institute of Medicine, University of Sri Lanka.

Sumathipala, A., Hewege, S., Hanwella, R., et al (2000) Randomized controlled trial of cognitive behaviour therapy for repeated consultations for medically unexplained complaints: a feasibility study in Sri Lanka. Psychological Medicine, 30, 747-757.

Sumathipala, A., Siribaddana, S. H. \& Bhugra, D. (2004) Culturebound syndromes: the story of dhat syndrome. British Journal of Psychiatry, 184, 200-209.

Thomas, K. B. (1987) General practice consultations: is there any point in being positive? BMJ, 294, 1200-1202.

Wessely, S. (1996) The rise of counseling and its effect on utilization of health care resources. BMJ, 313, 158-160.

World Health Organization (1992) The ICD-10 Classification of Mental and Behavioural Disorders: Clinical Descriptions and Diagnostic Guidelines. Geneva: WHO.

\section{MCQs}

1 In developing countries, the management of somatisation in primary care should include:

a excluding nutritional and infectious causes of complaints

b referral to psychiatric clinics

c cognitive-behavioural therapy

d psychodynamic therapy

e case management by a primary care practitioner.
2 In developing countries patients with medically unexplained symptoms characteristically:

a are overusers of healthcare facilities

b prefer to consult mental health specialists

c are very disabled by their symptoms

d are satisfied with numerous investigations carried out on them and the treatments they receive

e often have associated minor physical illness.

3 Patients with medically unexplained symptoms will gain long-term benefit from:

a discussing their symptoms, fears and concerns with as many family members as possible

$b$ repeated medical investigations as and when they become distressed by their symptoms

c referral to specialists to exclude all possible physical illnesses

d being relieved of their household responsibilities

e symptomatic medication.

4 The following statements are true of cognitivebehavioural therapy:

a the patient's behaviour is central to emotional, cognitive and physiological changes in their body

b it is of no benefit when medically unexplained symptoms are comorbid with another physical diagnosis

c patients should identify their own beliefs and assumptions as part of the treatment

d diary-keeping is an important part of the treatment

e challenging automatic thoughts is dangerous.

5 In cognitive-behavioural therapy:

a the number of sessions should be unlimited and decided by the patient

b the therapist should take sole responsibility for implementing the treatment

c educating the patient is a component

$\mathrm{d}$ response prevention is a part of the therapy

e restructuring dysfunctional cognitions will help to modify dysfunctional behaviours. 